\title{
Currents, Charges, and Near Fields of Cylindrical Antennas
}

\author{
Ronold W. P. King and Tai Tsun Wu
}

\section{Contribution From Gordon McKay Laboratory, Harvard University, Cambridge, Mass.}

(Received July 20, 1964; revised August 25, 1964)

\begin{abstract}
The commonly made assumption that the distribution of current along a dipole antenna is sinusoidal is examined critically together with the associated distribution of charge and the electromagnetic field. Measured distributions of current and charge are compared with those assumed in the sinusoidal theory. It is shown that the distributions of charge differ so greatly that it must be concluded that the electric field near and along the surface of the actual antenna is not well approximated by the near field obtained with the sinusoidal assumption.

A new approximate solution of the integral equation for the current in a center-driven cylindrical antenna is obtained in terms of simple trigonometric functions. It is shown that this and the associated distribution of charge are in much better agreement with measurements than results obtained from the sinusoidal assumption. Approximate expressions are then derived for the electromagnetic field near the antenna in a form that satisfies the boundary conditions.
\end{abstract}

\section{Introduction}

Studies of dipole antennas often postulate the current

$$
I_{z}(z)=I_{m} \sin k_{0}(h-|z|
$$

where $h$ is the half length of a perfectly conducting cylinder of radius $a$. The axis of the antenna coincides with the $z$-axis of the cylindrical coordinates $\rho, \theta, z ; k_{0}=2 \pi / \lambda=\omega / c$ where $c=\sqrt{\mu_{0} \epsilon_{0}}$ is the velocity of light. The distribution (1) has been used not only for far-field patterns, but to determine the near field and the admittance. Recently this procedure was applied to antennas in dissipative materials including magneto-ionic media in which the surface of the antenna may acquire a charge [Ament et al., 1964].

It is the purpose of this paper to examine the limitations of the sinusoidally distributed current and to derive an alternative simple distribution that is a better approximation.

\section{Sinusoidal Distribution and Its Implications}

The current (1) and the associated charge per unit length,

$$
q(z)=\frac{j}{\omega} \frac{d I_{z}(z)}{d z}=\mp \frac{j I_{m}}{c} \cos k_{0}(h-z), \quad z \gtrless 0
$$

are rigorous for a coaxial line that supports only the TEM mode and is terminated at $z=h$ in an ideal open circuit, $Z=\infty$ (fig. la). An ordinary open end (fig. lb) is not an ideal open circuit since as the end is approached the capacitance per unit length decreases to half the value which obtains at distances $h-z \gg b-a$ where $b$ is the inner radius of the outer conductor and $a$ is the radius of the inner conductor [King, 1955a]. As a consequence, $q(z)$ increases to double the value given by (2) at $z=h$ and the slope of the current curve departs from the sinusoid in (1). This capacitive end effect is confined to distances of the order of magnitude of $b-a$ [King, 1955b]. If the outer conductor is removed the inner conductor becomes an antenna (fig. lc) and the "end effect" extends for at least a quarter wavelength from the end. 


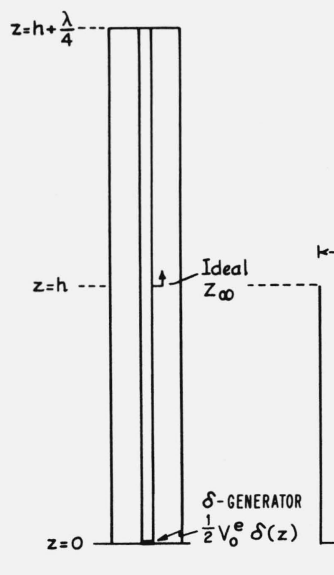

(a)

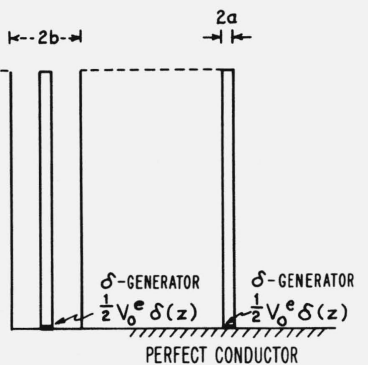

(b)
Figure 1. (a) Coaxial line terminated in ideal $\mathrm{Z}_{\infty}$ at $\mathrm{z}=\mathrm{h}$.

(b) Coaxial line terminated in open end.

(c) Base-driven monopole over perfectly conducting ground screen.

In a coaxial line in which (1) and (2) are accurate, the electric and magnetic fields are

$$
E_{\rho}(\rho, z)=\frac{q(z)}{2 \pi \epsilon_{0} \rho,} \quad B_{\theta}(\rho, z)=\frac{I(z)}{2 \pi v_{0} \rho}
$$

where $\nu_{0}=1 / \mu_{0}$. The scalar and vector potentials $\varphi(\rho, z)$ and $\mathbf{A}(\rho, z)$ satisfy the relations

$$
E_{\rho}(\rho, z)=\frac{-\partial \varphi(\rho, z)}{\partial \rho} ; \quad B_{\theta}(\rho, z)=\frac{-\partial A_{z}(\rho, z)}{\partial \rho} .
$$

It follows that

$$
\begin{aligned}
& \varphi(\rho, z)=-\int_{a}^{\rho} E_{\rho}(\rho, z) d \rho=\frac{-q(z)}{2 \pi \epsilon_{0}} \ln \frac{\rho}{a} \\
& A_{z}(\rho, z)=-\int_{a}^{\rho} B_{\theta}(\rho, z) d \rho=\frac{-I(z)}{2 \pi_{0}} \ln \frac{\rho}{a} .
\end{aligned}
$$

Note that

$$
E_{z}(\rho, z)=\frac{-\partial \varphi(\rho, z)}{\partial z}-j \omega A_{z}(\rho, z)=\frac{1}{2 \pi \epsilon_{0}}\left[\frac{\partial q(z)}{\partial z}+\frac{\mathrm{j} \omega}{c^{2}} I_{z}(z)\right] \ln \frac{\rho}{a}
$$

With (1) and (2)

$$
\frac{\partial q(z)}{\partial z}+\frac{\mathrm{j} \omega}{c^{2}} I_{z}(z)=0
$$

so that $E_{z}=0$ for all values of $\rho$.

The distributions (1) and (2) of current and charge in the coaixal line (fig. la) cannot be correct when the shield is removed to leave the base-driven monopole shown in figure lc since the boundary conditions are not the same. For the coaxial line $E_{z}=-V_{0}^{e} \delta(z)$ at $\rho=a, E_{z}=0$ at $\rho=b$; for the monopole $E_{z}=-V_{0}^{e} \delta(z)$ at $\rho=a$ and a radiation condition must be satisfied at infinity. (Note 
that the Dirac delta-function $\delta(z)$ has the properties $\delta(z)=0, z \neq 0 ; \delta(z)=\infty, z=0 ; \int_{-h}^{h} \delta(z) d z=1$.) If a sinusoidal current is postulated for the antenna it must be maintained by a more elaborate system of generators than a single delta-function $V_{n}^{e} \delta(z)$. Actually a continuous distribution of sources is required.

The electromagnetic field maintained by the sinusoidally distributed current (1) is [King, 1956a]

$$
\begin{aligned}
& B_{\theta}(\rho, z)=\frac{j I_{m}}{4 \pi \nu_{0} \rho}\left(e^{-j k_{0} r_{1 h}}+e^{-j k_{0} r_{2 h}}-2 \cos k_{0} h e^{-j k_{0} r_{0}}\right) \\
& E_{\rho}(\rho, z)=\frac{j I_{m} \zeta_{0}}{4 \pi \rho}\left(\frac{z-h}{r_{1 h}} e^{-j k_{0} r_{1 h}}+\frac{z+h}{r_{2 h}} e^{-j k_{0} r_{2 h}} \frac{2 z}{\dot{r}_{0}} \cos k_{0} h e^{-j k_{0} r_{0}}\right) \\
& E_{z}(\rho, z)=\frac{-j I_{m} \zeta_{0}}{4 \pi}\left(\frac{e^{-j k_{0} r_{1 h}}}{r_{1 h}}+\frac{e^{-j k_{0} r_{2 h}}}{r_{2 h}}-\frac{2}{r_{0}} \cos k_{0} h e^{-j k_{0} r_{0}}\right)
\end{aligned}
$$

where $r_{0}=\sqrt{z^{2}+\rho^{2}}, r_{1 h}=\sqrt{(h-z)^{2}+\rho^{2}}, r_{2 h}=\sqrt{(h+z)^{2}+\rho^{2}}$ and $\zeta_{0}=120 \pi$ ohms. These formulas have been derived from the vector and scalar potentials,

$$
A_{z}(\rho, z)=\frac{1}{4 \pi \nu_{0}} \int_{-h}^{h} I_{z}\left(z^{\prime}\right) \frac{e^{-j k_{0} r}}{r} d z^{\prime}, \quad \varphi(\rho, z)=\frac{1}{4 \pi \epsilon_{0}} \int_{-h}^{h} q\left(z^{\prime}\right) \frac{e^{-j k_{0} r}}{r} d z^{\prime}
$$

where $r=\sqrt{\left(z-z^{\prime}\right)^{2}+\rho^{2}}$ and where $I_{z}(z)$ and $q(z)$ are in (1) and (2), by means of the relations

$$
B_{\theta}(\rho, z)=\frac{-\partial A_{z}(\rho, z)}{\partial \rho}, \quad E_{\rho}(\rho, z)=\frac{-\partial \varphi(\rho, z)}{\partial \rho}, \quad E_{z}(\rho, z)=\frac{-\partial \varphi(\rho, z)}{\partial z}-j \omega A_{z}(\rho, z) .
$$

At a small radial distance $\rho$ given by $a \leqslant \rho \ll h, k_{0} a \leqslant k_{0} \rho \ll 1$, the approximations $r_{1 h} \doteq h-z$, $r_{2 h} \doteq h+z, r_{0} \doteq z$ may be made in the exponents. It follows that

$$
B_{\theta}(\rho, z) \doteq \frac{I_{m}}{2 \pi{ }^{\prime} \rho} \rho \sin k_{0}(h-|z|)=\frac{I_{z}(z)}{2 \pi \nu_{o} \rho}
$$

Thus, near the antenna, $B_{\theta}(\rho, z)$ is proportional to the current as in (3) for the coaxial line.

If short distances near $z=0, \pm h$ are excluded, and the same conditions are imposed on $\rho$,

$$
E_{\rho}(\rho, z) \doteq \mp \frac{j I_{m} \zeta_{0}}{2 \pi \rho} \cos k_{0}(h-z)=\frac{q(z)}{2 \pi \epsilon_{0} \rho}
$$

where the upper sign is for $z<0$, the lower for $z<0$. It is seen that near the antenna $E_{\rho}(\rho, z)$ is proportional to $q(z)$ as in (3) for the coaxial line.

Since at $\rho=a$ the surface densities of current and charge are $K_{z}(z)=I_{z}(z) / 2 \pi a$ and $\eta(z)=$ $q(z) / 2 \pi a$, it follows that at $\rho=a(12)$ and (13) reduce to the boundary condition for the tangential and the normal electric fields.

Although $B_{\theta}(\rho, z)$ and $E_{\rho}(\rho, z)$ are the same near and on the antenna as on the coaxial line when the currents in both are sinusoidal, this is not true of $E_{z}(\rho, z)$. On the coaxial line $E_{z}(a, z)=V_{0}^{e} \delta(z)$; on the antenna with an assumed sinusoidal current (9c) applies. However, it does not reduce to a simple form at $\rho=a$. Its magnitude at $\rho=a$ for antennas with $k_{0} h=\frac{\pi}{2}$ and $\pi$ and $\frac{a}{\lambda}=7.022 \times 10^{-3}$ is shown in figures $2 \mathrm{a}$ and $2 \mathrm{~b}$. Evidently, the distribution of $E_{z}(a, z)$ obtained from (9c) must be maintained along the antenna by a distribution of sources. Near the antenna where $\rho<<h$ and $k_{0} \rho<<1$, the general relation $\nabla \times \mathbf{E}=-j \omega \mathbf{B}$ gives

$$
\frac{\delta E_{z}(\rho, z)}{\delta z}=\frac{\delta E_{\rho}(\rho, z)}{\delta z}+j \omega B_{\theta}(\rho, z)=\frac{1}{2 \pi \rho}\left[\frac{1}{\epsilon_{0}} \frac{\delta q(z)}{\delta z}+\frac{j \omega}{\nu_{0}} I_{z}(z)\right] \doteq 0
$$



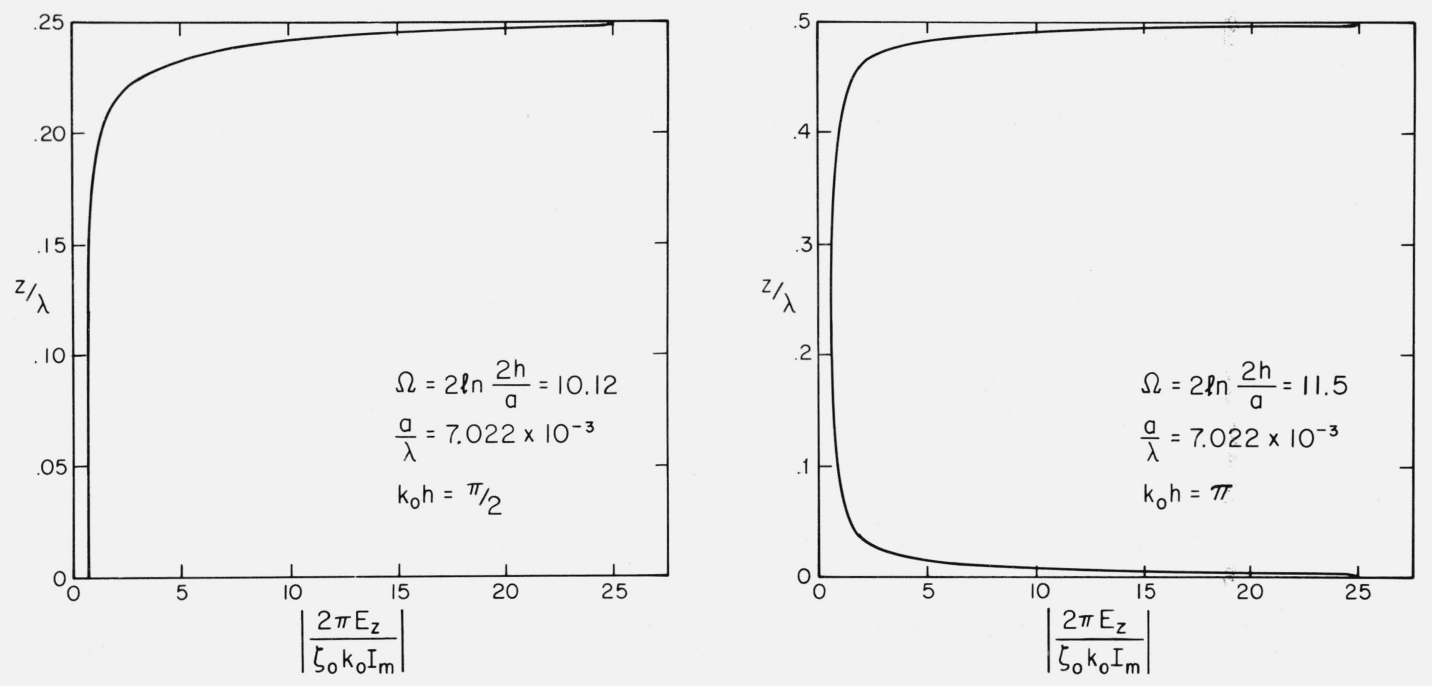

Figure 2. (a) Magnitude of $\mathrm{E}_{\mathrm{z}}(\rho, \mathrm{z})$ at $\rho=\mathrm{a}$ for an antenna with $\mathrm{k}_{0} \mathrm{~h}=\pi / 2$.

(b) Magnitude of $\mathrm{E}_{\mathrm{z}}(\rho, \mathrm{z})$ at $\rho=\mathrm{a}$ for an an. tenna with $\mathrm{k}_{0} \mathrm{~h}=\pi$.

This follows when (12) and (13) are substituted for $I_{z}(z)$ and $q(z)$. Near the antenna, where (12) and (13) are acceptable approximations, $E_{z}(\rho, z)$ is practically independent of $\rho$ and equal to the value at $\rho=a$. That is, $E_{z}(\rho, z) \doteq E_{z}(a, z)$.

A continuous distribution of sources along the antenna and the associated nonvanishing $E_{z}(a, z)$ require an outward radial component of the complex Poynting vector

$$
S_{\rho}(a, z)=-\frac{1}{2} \nu_{0} E_{z}(a, z) B_{\theta}^{*}(a, z)
$$

instead of the value

$$
S_{\rho}(a, z)=-\frac{1}{2} \nu_{0} E_{z}(a, z) B_{\theta}^{*}(a, z)=\frac{1}{2} \nu_{0} V_{0}^{e} B_{\theta}^{*}(a, z) \delta(z)
$$

which obtains on a perfectly conducting center-driven antenna. Note that with (3)

$$
P=\operatorname{Re} 2 \pi a \int_{-h}^{h} S_{\rho}(a, z) d z=\operatorname{Re} \frac{1}{2} V_{0}^{e} I_{z}^{*}(0)
$$

which is the total power supplied to and radiated from the antenna with a delta-function generator at its center. The total power radiated from the antenna with an assumed sinusoidally distributed current is

$P=\operatorname{Re} 2 \pi a \int_{-h}^{h} S_{\rho}(a, z) d z=-\operatorname{Re} 2 \pi a \int_{-h}^{h} \frac{1}{2} \nu_{0} E_{z}(a, z) B_{\theta}^{*}(a, z) d z \doteq-\operatorname{Re} \frac{1}{2} \int_{-h}^{h} E_{z}(a, z) I_{z}^{*}(z) d z$

where $E_{z}(a, z)$ and $B_{\theta}(a, z)$ are given by $(9 \mathrm{c})$ and $(9 \mathrm{a})$. 


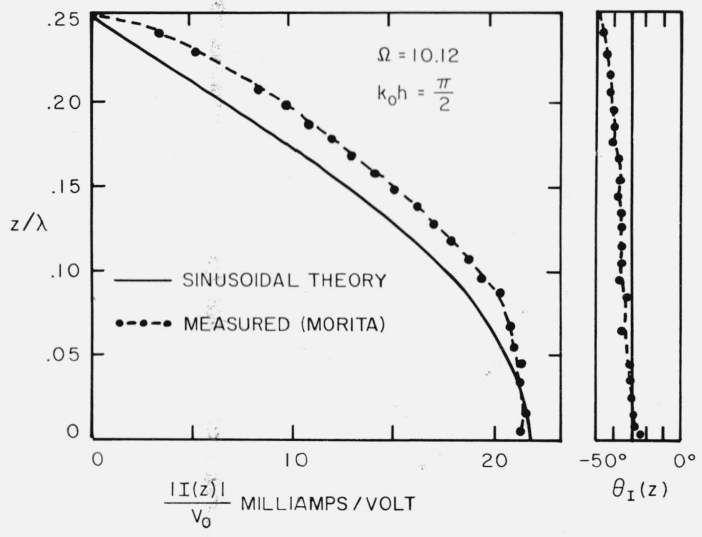

FigURE 3. Current along a cylindrical half-wave monopole $\left(\mathrm{k}_{0} \mathrm{~h}=\pi / 2, \mathrm{~h} / \lambda=1 / 4\right)$ over an image plane; $\Omega=10.12$.

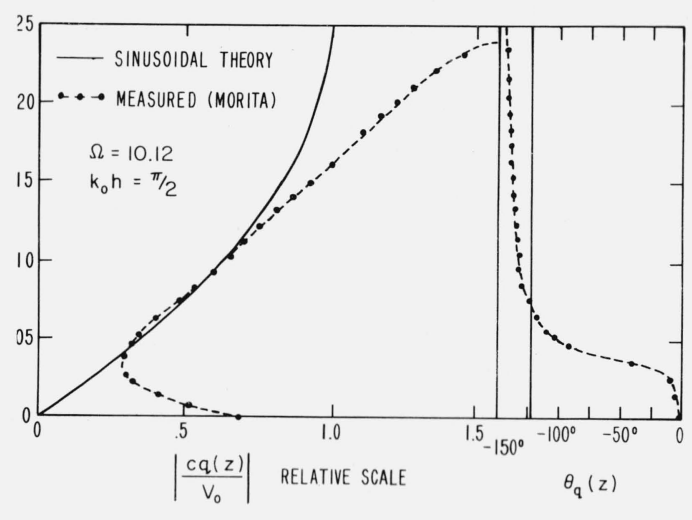

FIGURE 4. Distributions of charges along a half-wave dipole.

\section{Comparison With Experiment}

In figure 3 the current along a half-wave monopole is shown when $\Omega=2 \ln \frac{2 h}{a}=10.12$ or $\frac{h}{a} \doteq 78$. The corresponding curves for the cosinusoidal distribution (solid line) are in the form

$$
\frac{I_{z}(z)}{V_{0}^{e}}=\frac{\cos k_{0} z}{Z_{0}}=\frac{\cos k_{0} z}{\left|Z_{0}\right|} e^{-j \theta_{0}}
$$

where $Z_{0}=36.56+j 21.25=43.3 \exp \left(j 30.2^{\circ}\right) \Omega$. The general agreement between the measured values and the sinusoidal approximation is fair, with more current near the top of the actual antenna than is indicated by the cosine curve. Also the phase differs somewhat from the constant required by the sinusoidal theory. The large difference between the sinusoidal approximation and measurement in the distribution of charge along a half-wave dipole is shown in figure 4 where the measured amplitude and phase are in broken lines. The equation of continuity, $d I_{z}(z) / d z+j \omega q(z)=0$, when applied to (17) gives

$$
\frac{c q(z)}{V_{0}^{e}}=\frac{\sin k_{0} z}{\left|Z_{0}\right|} e^{-j\left(\theta_{0}+90^{\circ}\right)}
$$

The solid curves in figure 4 are for $\left|c q(z) / V_{0}^{e}\right|$ and $\theta_{q}=-\left(\theta_{0}+90^{\circ}\right)=-120.2^{\circ}$. The agreement between them and the measured amplitude and relative phase angle is quite poor. A large part of the charges actually present near the driving-point and the end of the antenna is not included in the cosine curve. The fact that the measured phase changes rapidly and greatly at some distance from the driving point is ignored by the sinusoidal theory.

Corresponding graphs for driven full-wave antennas are in figures 5 and 6 . Note that the sinusoidal theory fails completely near the driving point where instead of $I_{z}(0)=0, I_{z}(0)$ is actually nearly three-quarters of the maximum value. There is also more current in the upper half of the antenna than in the sinusoidal distribution and the phase angle, instead of independent of $z$ changes significantly along the entire antenna. The maximum current $I_{m}$ in figure 5 has been chosen so that the total powers radiated by the sinusoidal and the actual currents are equal. Specifically, $P / V_{0}^{e^{2}}=G_{0}=\left|I_{0} / V_{0}\right|^{2} R_{0}=1.023 \times 10^{-3}$ mhos is the power per unit voltage squared supplied to the actual antenna. The power radiated by the sinusoidal current is $I_{m}^{2} R_{m}^{e}=199.1 I_{m}^{2}$. This is equal to $1.023 \times 10^{-3}$ when $I_{m}=2.26 \times 10^{-3} V_{0}^{e}$. 


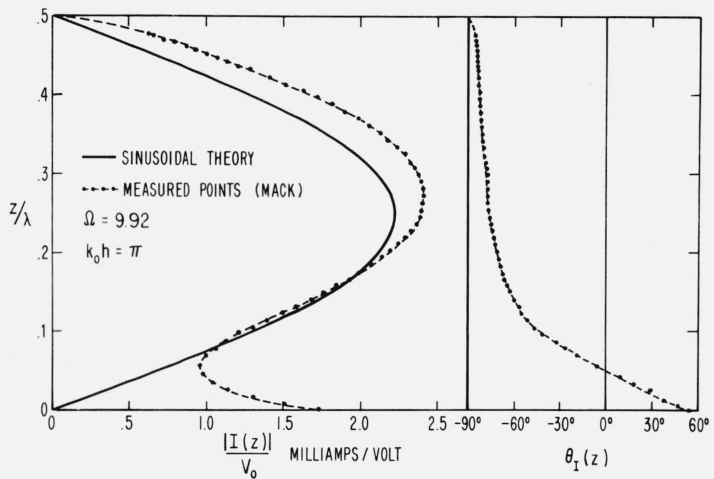

FIGURE 5. Distributions of current and charge alol.g center-driven full-wave antenna; $\mathrm{k}_{0} \mathrm{~h}=\pi, \Omega=9.92$.

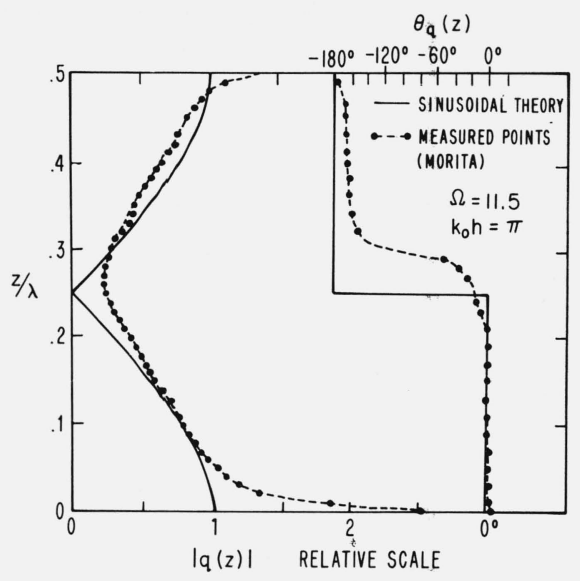

FIGURE 6. Distributions of current and charge along center-driven full-wave antenna; $\mathrm{k}_{0} \mathrm{~h}=\pi, \Omega=11.5$.

The distribution of charge along the full-wave dipole (fig. 6) varies almost linearly with distance so that large concentrations of charge near the driving point and at the ends are not included in a cosine curve. The abrupt reversal in phase at $z=0.25 \lambda$, which is predicted by the sinusoidal theory, is replaced by a rapid reversal near $z \doteq 0.3 \lambda$.

In view of the reasonable agreement between the sinusoidal approximation and actual distributions in some respects and the large differences in others, the question arises as to how accurately such quantities as the far and near field, the admittance and the radiated power can be determined from the sinusoidal assumption.

In the radiation field $\left(k_{0} r>>1\right)$ the electric and magnetic vectors differ only by a constant coefficient since $E_{\odot}^{r}=c B_{\Phi}^{r}$. Moreover, both depend directly on the integral

$$
\int_{-h}^{h} I_{z}\left(z^{\prime}\right) e^{j k_{0} z^{\prime} \cos \odot} \sin \odot d z^{\prime}
$$

which is insensitive to the details of the distribution of current and is independent of the charges. Hence the far field patterns of the actual currents (figs. 3 and 5) should be in reasonable agreement with the patterns of the sinusoidal currents. (In the case of longer antennas $\left(h>\frac{\lambda}{2}\right)$ the patterns involve minor maxima and minima that depend on rather precise relations between the phases and amplitudes of the currents along the antenna, so that greater divergences between the measurements and the sinusoidal theory may be anticipated.)

When the currents are normalized and the antennas and the surrounding medium are lossless, the total power radiated is the same for both distributions. The real part of the integral of the normal component of $\mathbf{S}=\frac{1}{2} \nu_{0} \mathbf{E} \times \mathbf{B}^{*}$ over any surface which completely encloses the generator is, therefore, also the same for the two distributions.

Whereas the magnitude of the magnetic field of the sinusoidally distributed current is a fair approximation of the magnetic field of an actual cylindrical antenna at all points (since the sinusoidal distribution is a reasonable approximation of the current), this is true of the electric field only in the far zone. The electric field near a sinusoidally distributed current differs greatly from that of a center-driven cylindrical antenna since $E_{\rho}$ near the antenna depends on $q(z)$ and it has been shown that the cosinusoidal approximation for $q(z)$ is in poor agreement with the actual distribution. Moreover, since a sinusoidally distributed current cannot be generated by a single source along a perfectly conducting cylinder, a continuous distribution of generators is required to maintain an impressed tangential electric field along the entire antenna. A resultant nonvanishing tangential electric field then obtains and this is not a good approximation of $\boldsymbol{E}_{z}=0$ along a perfectly conducting cylinder. 
It is not actually possible to define a driving-point impedance for an antenna with a sinusoidally distributed current since there is no single localized generator. A quantity that is dimensionally an impedance may be introduced in terms of the total power defined in (16b) and the current at the center of the antenna. Thus,

$$
Z_{0}=\frac{2 P}{I_{z}(0) I_{z}^{*}(0)}
$$

Since the generator is distributed along the entire antenna (20) is not a driving-point impedance. The driving-point impedance of the cylindrical antenna is

$$
Z_{0}=V_{0}^{e} / I_{z}(0)
$$

It is not surprising that (20) for the sinusoidal theory and (21) for the center-driven cylindrical antenna differ greatly except in special cases. $Z_{0}$ in (20) is a function of $k_{0} h$ only, whereas in (2l) $Z_{0}$ depends on both $k_{0} h$ and $\frac{h}{a}$. When $k_{0} h=\frac{\pi}{2},(20)$ gives $Z_{0}=73.1+j 42.5 \Omega$ or $Y_{0}=(10.22-j 5.94)$ $\times 10^{-3}$ mhos, whereas (21) ranges from $Z_{0}=78.5+j 43.6$ when $\frac{h}{a} \sim 11,000$ to $Z_{0}=108+j 36$ when $\frac{h}{a}$ $\sim 16.5$. When $k_{0} h=\pi,(20)$ gives $Z_{0}=\infty$ and $Y_{0}=0,(21)$ gives $Z_{0}=2674-j 2630$ when $\frac{h}{a} \sim 11,000$ and $Z_{0}=264-j 436$ when $\frac{h}{a} \sim 75$. Actually, (20) with a sinusoidal current is a reasonable approximation of (21) for a highly conducting cylinder only when $k_{0} h$ is near $\frac{\pi}{2}$ and $\frac{h}{a} \geqslant 10,000$. Since the driving-point impedance depends critically on both the electric and the magnetic fields at the surface of the antenna and since the sinusoidal theory provides a fair approximation of the magnetic field but not of the electric field, general agreement cannot be expected.

\section{Integral Equation and Its Solution}

The measured distribution of current, the impedance, and especially the distribution of charge and the near field of a center-driven cylindrical antenna differ significantly from the quantities obtained when a sinusoidally distributed current is assumed. More accurate solutions of the integral equation for the current have been derived by iteration [Hallén, 1938; King and Harrison, 1943; King 1956b], by variational methods [King, 1956b; Storer, 1951; Tai, 1950] and by judicious separation and fitting [King, 1959]. It is the purpose of this study to derive a new solution which combines considerable accuracy with reasonable simplicity for cylindrical antennas with $k_{0} h \leqslant 5 \pi / 4$.

The differential equation for the vector potential $A_{z}(z)$ at $\rho=a$ on a cylindrical antenna center driven by a delta-function generator is [King, 1956b]

$$
\left(\frac{\partial^{2}}{\partial z^{2}}+k_{0}^{2}\right) A_{z}(z)=-j \frac{k_{0}^{2}}{\omega} V_{0}^{e} \delta(z)
$$

where the time dependence is $e^{j \omega t}$. It is assumed that $k_{0} a \ll 1, a \ll h . \quad A_{z}(z)=A_{R}(z)+j A_{I}(z)$ is given approximately by

$$
A_{z}(z)=\frac{1}{4 \pi \nu_{0}} \int_{-h}^{h} I_{z}\left(z^{\prime}\right) K\left(z, z^{\prime}\right) d z^{\prime}
$$

where

$$
K\left(z, z^{\prime}\right)=e^{-j k o r} / r, \quad r=\sqrt{\left(z-z^{\prime}\right)^{2}+a^{2}} .
$$


The real and imaginary parts of the vector potential are

$$
\begin{aligned}
& A_{R}(z)=\frac{k_{0}}{4 \pi \nu_{0}} \int_{-h}^{h} I_{z}\left(z^{\prime}\right) \frac{\cos k_{0} r}{k_{0} r} d z^{\prime} \\
& A_{I}(z)=\frac{-k_{0}}{4 \pi \nu_{0}} \int_{-h}^{h} I_{z}\left(z^{\prime}\right) \frac{\sin k_{0} r}{k_{0} r} d z^{\prime} .
\end{aligned}
$$

The solution of (22) is readily combined with (24) to obtain the integral equation for the current [King, 1956b]:

$$
\int_{-h}^{h} I_{z}\left(z^{\prime}\right) K\left(z, z^{\prime}\right) d z^{\prime}=\frac{j 4 \pi}{\zeta_{0}}\left[C_{1} \cos k_{0} z+\frac{1}{2} V_{0}^{e} \sin k_{0}|z|\right]
$$

where $\zeta_{0}=\sqrt{\mu_{0} / \epsilon_{0}}=1 / \sqrt{\nu_{0} \epsilon_{0}} \doteq 120 \pi$ ohms.

Approximate formal solutions of (26) have been described [King, 1956b] and accurate results for particular cases can be obtained by numerical methods. In order to derive an analytically simple and reasonably accurate solution for $I_{z}(z)$ use may be made of the quite different properties of the kernels in $(25 \mathrm{a}, \mathrm{b})$. In particular, the kernel in (25a) is very large at $z^{\prime}=z$ where $r=a$, the kernel in (25b) is not. Specifically, at $z^{\prime}=z$

$$
\begin{aligned}
& \frac{\cos k_{0} r}{k_{0} r}=\frac{\cos k_{0} a}{k_{0} a} \doteq \frac{1}{k_{0} a}>>1 \\
& \frac{\sin k_{0} r}{k_{0} r}=\frac{\sin k_{0} a}{k_{0} a} \doteq 1 .
\end{aligned}
$$

Graphs of the two kernels in figure 7 show that $A_{R}(z)$ must vary with $z$ like $I_{z}(z)$. However, since

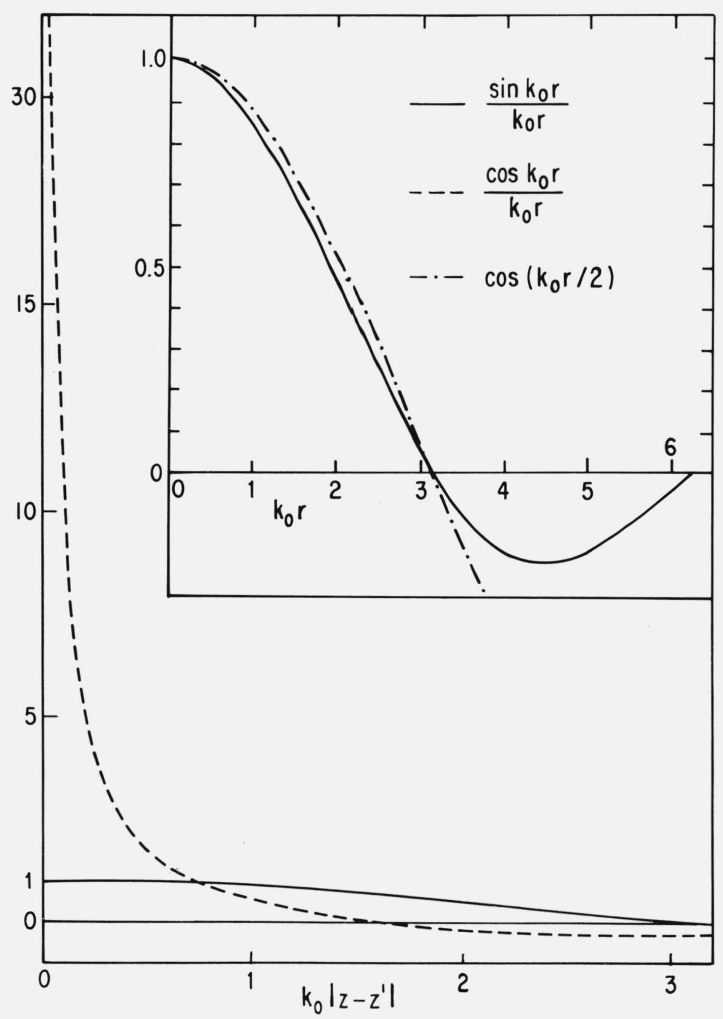

FiguRE 7. The functions $\frac{\sin \mathrm{k}_{0} \mathrm{r}}{\mathrm{k}_{0} \mathrm{r}}, \frac{\cos \mathrm{k}_{0} \mathrm{r}}{\mathrm{k}_{0} \mathrm{r}}$ and $\cos \left(\mathrm{k}_{0} \mathrm{r} / 2\right)$. 
$I_{z}(h)=0$ whereas $A_{R}(h)$ is small but finite, the difference $A_{R}(z)-A_{R}(h)$ should be approximately proportional to $I_{z}(z)$. That is

$$
4 \pi \nu_{0}\left[A_{R}(z)-A_{R}(h)\right]=\Psi(z) I_{z}(z) \doteq \Psi_{d R} I(z)
$$

where $\Psi_{d R}$ is the approximately constant value of $\Psi(z)$ defined at a suitable reference value of $z$.

The graph of $\left(\sin k_{0} r\right) / k_{0} r$ in figure 7 shows that contributions to $A_{I}(z)$ come almost equally from elements of current over a large range of $\left|z^{\prime}-z\right|$. Hence, an approximation of $A_{I}(z)$ can be obtained from

$$
\frac{\sin k_{0} r}{k_{0} r}=\frac{2 \sin \frac{1}{2} k_{0} r \cos \frac{1}{2} k_{0} r}{k_{0} r} \doteq \cos \frac{1}{2} k_{0} r
$$

over the range $k_{0} r \leqslant \pi$ as shown in figure 7. With (29), (25b) may be expressed as follows:

$$
A_{I}(z) \doteq \frac{-k_{0}}{4 \pi \nu_{0}} \int_{0}^{h} I_{z}\left(z^{\prime}\right)\left[\cos \frac{1}{2} k_{0} r_{1}+\cos \frac{1}{2} k_{0} r_{2}\right] d z^{\prime}
$$

where $r_{1}=\sqrt{\left(z-z^{\prime}\right)^{2}+a^{2}} \doteq\left|z-z^{\prime}\right|, r_{2}=\sqrt{\left(z+z^{\prime}\right)^{2}+a^{2}} \doteq\left|z+z^{\prime}\right|$. The trigonometric functions can be combined to give

$$
A_{I}(z) \doteq \frac{-2 k_{0}}{4 \pi \nu_{0}} \cos \frac{1}{2} k_{0} z \int_{0}^{h} I_{z}\left(z^{\prime}\right) \cos \frac{1}{2} k_{0} z^{\prime} d z^{\prime}
$$

It follows that since

$$
A_{I}(z) \doteq A_{I}(0) \cos \frac{1}{2} k_{0} z
$$

where $A_{I}(0)$ is a constant, $A_{I}(\mathrm{z})$ is independent of the distribution of current. Also

$$
A_{I}(z)-A_{I}(h) \doteq A_{I}(0) F_{0 z}^{\prime}
$$

where

$$
F_{0 z}^{\prime}=\cos \frac{1}{2} k_{0} z-\cos \frac{1}{2} k_{0} h .
$$

An integral equation that involves the vector potential difference [King, 1959] is obtained from a rearrangement of (26). It is

$$
\int_{-h}^{h} I_{z}\left(z^{\prime}\right) K_{d}\left(z, z^{\prime}\right) d z^{\prime}=\frac{j 4 \pi}{\zeta_{0} \cos k_{0} h}\left[\frac{1}{2} V_{0}^{e} M_{0 z}+U F_{0 z}\right]
$$

where

$$
\begin{gathered}
M_{0 z}=\sin k_{0}(h-|z|) \\
F_{0 z}=\cos k_{0} z-\cos k_{0} h \\
K_{d}\left(z, z^{\prime}\right)=K\left(z, z^{\prime}\right)-K\left(h, z^{\prime}\right)
\end{gathered}
$$

and

$$
U_{k}=\frac{-j \omega}{k_{0}} A_{z}(h)=\frac{-j \zeta_{0}}{4 \pi} \int_{-h}^{h} I_{z}\left(z^{\prime}\right) K\left(h, z^{\prime}\right) d z^{\prime}
$$


The properties, (28) and (32), of the integral on the left in (33) suggest the distribution of current

$$
I_{z}(z)=I_{V}(z)+I_{U}(z)+I_{D}(z)
$$

where

$$
I_{V}(z) \doteq I_{V} M_{0 z}, \quad I_{U}(z) \doteq I_{U} F_{0 z}, \quad I_{D}(z) \doteq I_{D} F_{0 z}^{\prime}
$$

and $I_{V}, I_{U}$, and $I_{D}$ are undetermined constant coefficients.

If (38) is substituted in the integral in (33) and use is made of (28) and (32) the following relations are obtained:

$$
\begin{aligned}
& \int_{-h}^{h} I_{V}\left(z^{\prime}\right) K_{d R}\left(z, z^{\prime}\right) d z^{\prime} \doteq I_{V}(z) \Psi_{d R} \\
& \int_{-h}^{h} I_{U}\left(z^{\prime}\right) K_{d R}\left(z, z^{\prime}\right) d z^{\prime} \doteq I_{U}(z) \Psi_{d U R} \\
& \int_{-h}^{h} I_{D}\left(z^{\prime}\right) K_{d}\left(z, z^{\prime}\right) d z^{\prime} \doteq I_{D}(z)\left[\Psi_{d D R}+j \Psi_{d D I}\right]=I_{D}(z) \Psi_{d D} \\
& \int_{-h}^{h} I_{V}\left(z^{\prime}\right) K_{d I}\left(z, z^{\prime}\right) d z^{\prime} \doteq\left(I_{V} / I_{D}\right) I_{D}(z) \Psi_{d I} \\
& \int_{-h}^{h} I_{U}\left(z^{\prime}\right) K_{d I}\left(z, z^{\prime}\right) d z^{\prime} \doteq\left(I_{U} / I_{D}\right) I_{D}(z) \Psi_{d U I} .
\end{aligned}
$$

Similarly, if (38) is substituted in (37)

$$
\int_{-h}^{h} I_{z}\left(z^{\prime}\right) K\left(h, z^{\prime}\right) d z^{\prime}=I_{V} \Psi_{V}(h)+I_{U} \Psi_{U}(h)+I_{D} \Psi_{D}(h) .
$$

Explicit formulas for the several $\Psi$ functions are in appendix A.

With (38)-(41) equation (33) is reduced to an algebraic equation in $M_{0 z}, F_{0 z}$ and $F_{0 z}^{\prime}$. However, since approximations have been made, it is preferable to make the substitution in the differential equation (19) in the following equivalent form:

$$
4 \pi \nu_{0}\left(\frac{d^{2}}{d z^{2}}+k_{0}^{2}\right)\left[A_{z}(z)-A_{z}(h)\right]=\frac{-j 4 \pi k_{0}}{\zeta_{0}} V_{0}^{e} \delta(z)-4 \pi \nu_{0} k_{0}^{2} A_{z}(h)
$$

With (24a, b) and (38)-(40e) it follows that:

$$
4 \pi \nu_{0}\left[A_{z}(z)-A_{z}(h)\right]=I_{V} \Psi_{d R} M_{0 z}+I_{U} \Psi_{d U R} F_{0 z}+\left(j I_{V} \Psi_{d I}+j I_{U} \Psi_{d U I}+I_{D} \Psi_{d D}\right) F_{0 z}^{\prime} .
$$

Differentiation and formation of the left side in (42) results in

[Left side of (42)] $=-2 I_{V} \Psi_{d R} k_{0} \cos k_{0} h \delta(z)-I_{U} \Psi_{d U R} k_{0}^{2} \cos k_{0} h$

$$
-\left(j I_{V} \Psi_{d \Gamma}+j I_{U} \Psi_{d U \Gamma}+I_{D} \Psi_{d D}\right) k_{0}^{2}\left(\cos \frac{1}{2} k_{0} h-\frac{3}{4} \cos \frac{1}{2} k_{0} z\right)
$$

With (41),

$$
[\text { Right side of }(42)]=\frac{-j 4 \pi k_{0}}{\zeta_{0}} V_{0}^{e} \delta(z)-k_{0}^{2}\left[I_{V} \Psi_{V}(h)+I_{U} \Psi_{U}(h)+I_{D} \Psi_{D}(h)\right]
$$


When (44a) and (44b) are equated, the coefficients of $\delta(z)$ must be equal. The resulting equation defines $I_{V}$ as follows:

$$
I_{V}=\frac{j 2 \pi V_{0}^{e}}{\zeta_{0} \Psi_{d R} \cos k_{0} h}
$$

The remaining equation reveals the approximate nature of the solution since the left side is a function of $z$, the right side a constant. However, within the restriction $k_{0} h \leqslant 5 \pi / 4$, the slowly varying function $\cos \frac{1}{2} k_{0} z$ can contribute only a small error in the distribution of current if the equation is satisfied at $z= \pm h$ and $z=0$. In this way two equations are obtained that determine $I_{U}$ and $I_{D}$ :

$$
\begin{gathered}
I_{U} C_{U}+I_{D} C_{D}=I_{V} C_{V} \\
I_{U} E_{U}+I_{D} E_{D}=I_{V} E_{V}
\end{gathered}
$$

where

$$
\begin{aligned}
& C_{U}=-\Psi_{d U R} \cos k_{0} h+j \Psi_{d U I}\left(\frac{3}{4}-\cos \frac{1}{2} k_{0} h\right)+\Psi_{U}(h) \\
& C_{D}=\Psi_{d D}\left(\frac{3}{4}-\cos \frac{1}{2} k_{0} h\right)+\Psi_{D}(h) \\
& C_{V}=-\left[j \Psi_{d I}\left(\frac{3}{4}-\cos \frac{1}{2} k_{0} h\right)+\Psi_{V}(h)\right] \\
& E_{U}=-\Psi_{d U R} \cos k_{0} h-j \frac{1}{4} \Psi_{d U I} \cos \frac{1}{2} k_{0} h+\Psi_{U}(h) \\
& E_{D}=-\frac{1}{4} \Psi_{d D} \cos \frac{1}{2} k_{0} h+\Psi_{D}(h) \\
& E_{V}=j \frac{1}{4} \Psi_{d I} \cos \frac{1}{2} k_{0} h-\Psi_{V}(h) .
\end{aligned}
$$

The equations $(46 \mathrm{a}, \mathrm{b})$ are readily solved in the form $I_{U}=T_{U} I_{V}, I_{D}=T_{D} I_{V}$, where

$$
\begin{aligned}
& T_{U}=\frac{C_{V} E_{D}-C_{D} E_{V}}{C_{U} E_{D}-C_{D} E_{U}}=Q^{-1}\left[\Psi_{d D} \Psi_{V}(h)-j \Psi_{d I} \Psi_{D}(h)\right] \\
& T_{D}=\frac{C_{U} E_{V}-C_{V} E_{U}}{C_{U} E_{D}-C_{D} E_{U}}=-j Q^{-1}\left\{\Psi_{d I}\left[\Psi_{d U R} \cos k_{0} h-\Psi_{U}(h)\right]+\Psi_{d U I} \Psi_{V}(h)\right\}
\end{aligned}
$$

with

$$
Q=\Psi_{d D}\left[\Psi_{d U R} \cos k_{0} h-\Psi_{U}(h)\right]+j \Psi_{d U I} \Psi_{D}(h)
$$

\section{Distribution of Current and Admittance}

With the amplitude coefficients $I_{V}, I_{U}$, and $I_{D}$ determined the approximate distribution of current is

$$
I_{z}(z)=\frac{j 2 \pi V_{0}^{e}}{\delta_{0} \Psi_{d R} \cos k_{0} h}\left\{\sin k_{0}(h-z)+T_{U}\left(\cos k_{0} z-\cos k_{0} h\right)+T_{D}\left(\cos \frac{1}{2} k_{0} z-\cos \frac{1}{2} k_{0} h\right)\right\}
$$


for $z \geqslant 0$. Note that $I_{z}(-z)=I_{z}(z)$. This formula differs by the term in $T_{D}$ from an earlier approximation [King, 1959]. It more accurately represents the distribution of current especially at some distance from the driving point. (Note, however, that the added term is strictly a self-term that in no way alters the contributions to the shifted cosine term by coupled antennas in arrays.) The admittance of the antenna is

$$
Y_{0}=\frac{j 2 \pi}{\delta_{0} \Psi_{d R} \cos k_{0} h}\left\{\sin k_{0} h+T_{U}\left(1-\cos k_{0} h\right)+T_{D}\left(1-\cos \frac{1}{2} k_{0} h\right)\right\} .
$$

The distribution of charge is

$$
q(z)=\frac{2 \pi \epsilon_{0} V_{0}^{e}}{\psi_{d R} \cos k_{0} h}\left\{\cos k_{0}(h-z)+T_{U} \sin k_{0} z+\frac{1}{2} T_{D} \sin \frac{1}{2} k_{0} z\right\} z \geqslant 0 .
$$

Note that $q(-z)=-q(z)$.

When $k_{0} h$ is near $\frac{\pi}{2},(49)$ and (50) become indeterminate and alternative forms are more convenient. By a simple rearrangement (49) becomes

$I_{z}(z)=\frac{-j 2 \pi V_{0}^{e}}{\delta_{0} \Psi_{d R}}\left\{\left(\sin k_{0} z-\sin k_{0} h\right)-T_{U}^{\prime}\left(\cos k_{0} z-\cos k_{0} h\right)-T_{D}^{\prime}\left(\cos \frac{1}{2} k_{0} z-\cos \frac{1}{2} k_{\theta} h\right\} z \geqslant 0\right.$

where

$$
T_{U}^{\prime}=-\frac{T_{U}+\sin k_{0} h}{\cos k_{0} h}, \quad T_{D}^{\prime}=\frac{T_{D}}{\cos k_{0} h}
$$

Explicit formulas are in appendix B. These show that $T_{U}^{\prime}$ and $T_{D}^{\prime}$ are finite when $k_{0} h=\frac{\pi}{2}$. The admittance corresponding to $(52 \mathrm{a})$ is

$$
Y_{0}=\frac{-j 2 \pi}{\delta_{0} \Psi_{d R}}\left\{-\sin k_{0} h+T_{U}^{\prime}\left(1-\cos k_{0} h\right)-T_{D}^{\prime}\left(1-\cos k_{0} h\right)\right\}
$$

The charge distribution is

$$
q(z)=\frac{2 \pi \epsilon_{0} V_{0}^{e}}{\Psi_{d R}}\left\{\cos k_{0} z-T_{U}^{\prime} \sin k_{0} z+\frac{1}{2} T_{D}^{\prime} \sin \frac{1}{2} k_{0} z\right\}, z \geqslant 0 .
$$

When the antenna is electrically short so that $k_{0} h<1$,

$$
\begin{gathered}
I_{z}(z) \doteq \frac{j 2 \pi V_{0}^{e}}{\delta_{0} \Psi_{d R}}\left[k_{0} h\left(1-\frac{z}{h}\right)+\frac{1}{2} k_{0}^{2} h^{2} T\left(1-\frac{z^{2}}{h^{2}}\right)\right] ; z \geqslant 0 \\
q(z)=\frac{2 \pi \epsilon_{0} V_{0}^{e}}{\Psi_{d R}}\left[1+T k_{0} z\right] ; z \geqslant 0
\end{gathered}
$$

where $T=T_{U}+T_{D} / 4$.

Numerical computations have been made for an antenna with $\frac{a}{\lambda}=7.022 \times 10^{-3}$ for which extensive measurements are available [Mack, 1964]. When $k_{0} h=\pi / 2$ with $\Omega=8.58$ and $k_{0} h=\pi$ with $\Omega=9.92$ the following quantities apply:

$$
\begin{aligned}
& k_{0} h=\frac{\pi}{2}, \quad \psi_{d R}=6.218, \quad T_{U}^{\prime}=3.085+j 3.581, \quad T_{D}^{\prime}=1.061+j 0.025 \\
& k_{0} h=\pi, \quad \psi_{d R}=5.737, \quad T_{U}=-0.117+j 0.114, \quad T_{D}=-0.106+j 0.108
\end{aligned}
$$


The corresponding currents, admittances, and charges per unit length are, for $k_{0} h=\frac{\pi}{2}$,

$$
\begin{gathered}
\frac{I_{z}(z)}{V_{0}^{e}}=\left\{9.597 \cos k_{0} z-0.067 \cos \frac{1}{2} k_{0} z+0.047-j\left[2.680\left(\sin k_{0} z-1\right)+8.269 \cos k_{0} z\right.\right. \\
Y_{0}=(9.577-j 4.756) \times 10^{-3}, \quad Z_{0}=83.76+j 41.60 \\
\frac{c q(z)}{V_{0}^{e}}=\left\{2.680 \cos k_{0} z-8.269 \sin k_{0} z+1.422 \sin \frac{1}{2} k_{0} z-j\left[9.597 \sin k_{0} z\right.\right. \\
\left.\left.-0.033 \sin \frac{1}{2} k_{0} z\right]\right\} \times 10^{-3} .
\end{gathered}
$$

For $k_{0} h=\pi$ they are

$$
\begin{gathered}
\frac{I_{z}(z)}{V_{0}^{e}}=\left\{0.331\left(\cos k_{0} z+1\right)+0.314 \cos \frac{1}{2} k_{0} z-j\left[2.905 \sin k_{0} z-0.340\left(\cos k_{0} z+1\right)\right.\right. \\
Y_{0}=(0.976+j 0.988) \times 10^{-3}, \quad Z_{0}=506.0-j 512.2 \\
\frac{c q(z)}{V_{0}^{e}}=-\left\{\left[0.331 \sin k_{0} z+0.157 \sin \frac{1}{2} k_{0} z\right]+j\left[2.905 \cos k_{0} z+0.340 \sin k_{0} z\right.\right. \\
\left.\left.+0.154 \sin \frac{1}{2} k_{0} z\right]\right\} \times 10^{-3} .
\end{gathered}
$$

Graphs of $I_{z}(z) / V_{0}^{e}=\left[I_{z}^{\prime \prime}(z)+j I_{z}^{\prime}(z)\right] / V_{0}^{e}$ are in figures 8 and 10 together with the measured results of Mack [1959]. Corresponding curves of $c q(z) / V_{0}^{e}=\left(j c / V_{0}^{e}\right)\left[q^{\prime \prime}(z)+j q^{\prime}(z)\right]$ are in figures 9 and 11. These were obtained from the slopes of the measured curves of the currents. The agreement between the approximate theory and experiment both for currents and charges indicates significant improvement over the sinusoidal theory.

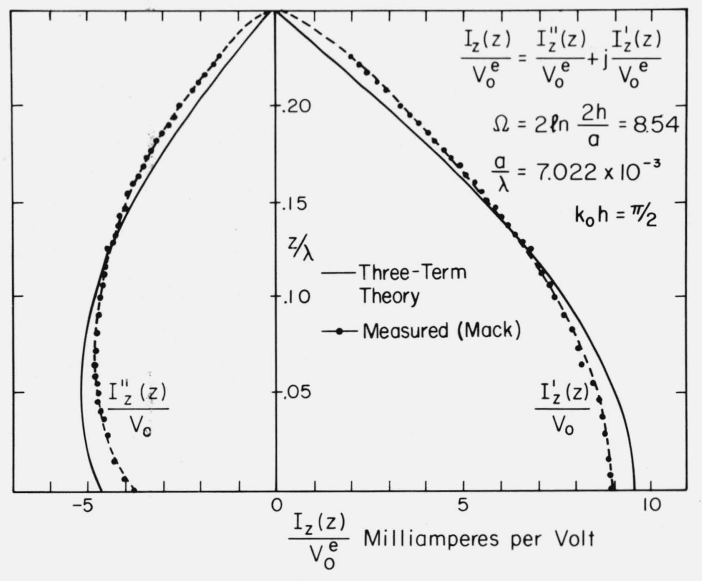

FIGURE 8. $\mathrm{I}_{\mathrm{z}}(\mathrm{z}) / \mathrm{V}_{0}^{\mathrm{e}}$ for an antenna with $\mathrm{k}_{0} \mathrm{~h}=\pi / 2, \Omega=8.54$.

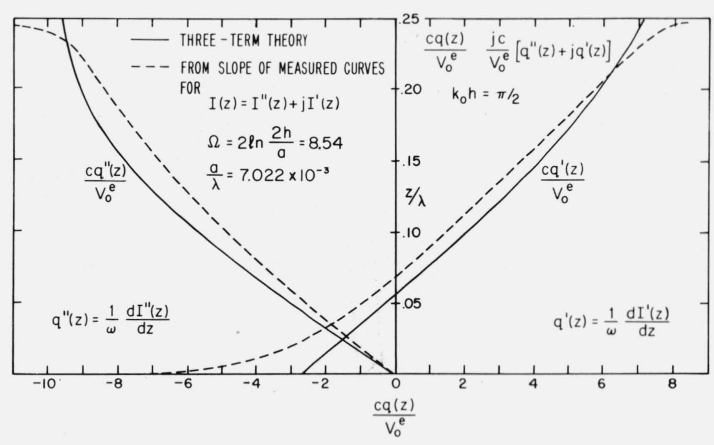

FigURE 9. $\operatorname{cq}(\mathrm{z}) / \mathrm{V}_{0}^{\mathrm{e}}$ for an antenna with $\mathrm{k}_{0} \mathrm{~h}=\pi / 2, \Omega=8.54$. 

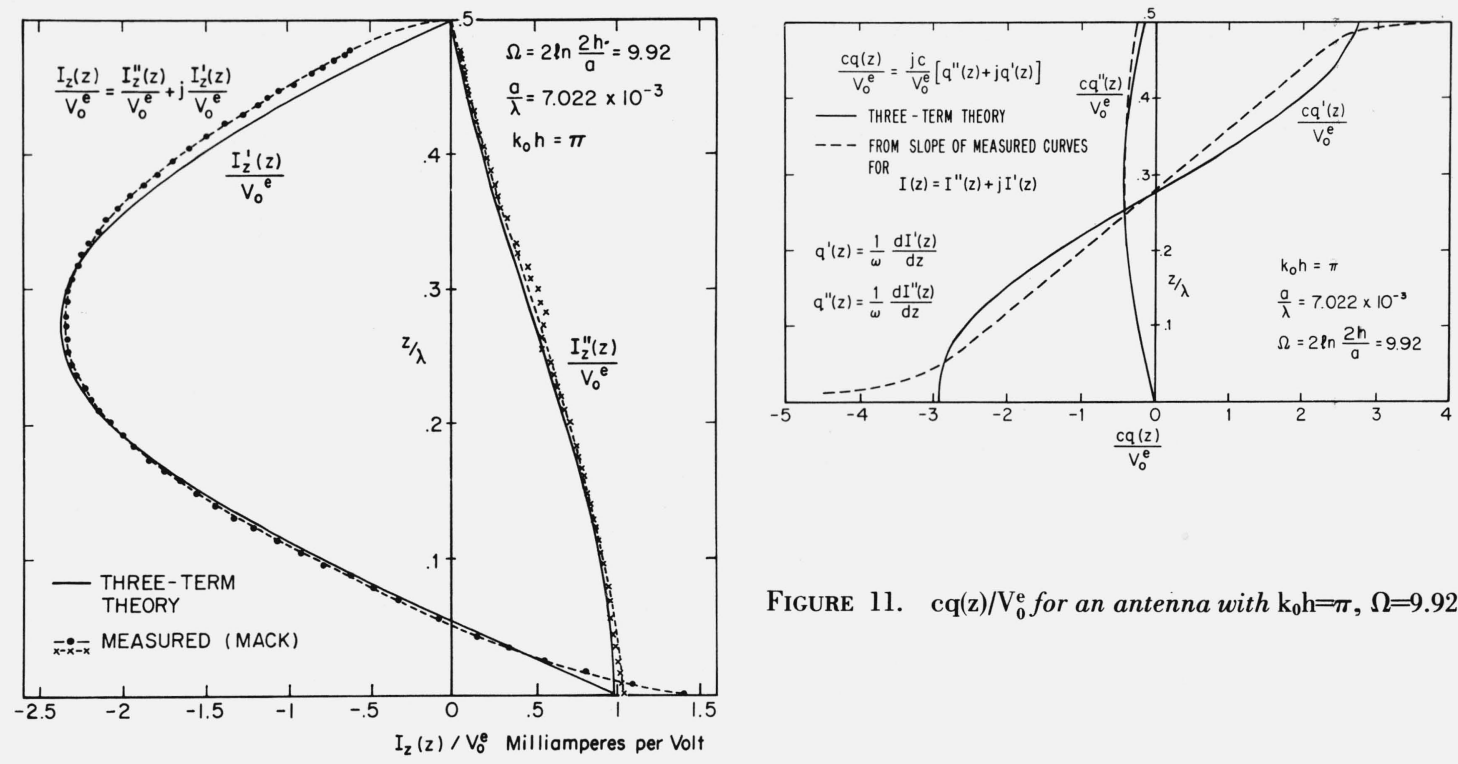

FIGURE 11. cq(z)/ $\mathrm{V}_{0}^{\mathrm{e}}$ for an antenna with $\mathrm{k}_{0} \mathrm{~h}=\pi, \Omega=9.92$.

FIGURE 10. $\mathrm{I}_{\mathrm{z}}(\mathrm{z}) / \mathrm{V}_{0}^{\mathrm{e}}$ for an antenna with $\mathrm{k}_{0} \mathrm{~h}=\pi, \Omega=9.92$.

\section{Electromagnetic Field}

If the three-term approximations of the currents and charges are substituted in $(10 a, b)$ and these are then used in (11) expressions are obtained for the electromagnetic field that are better approximations than $(9 a-c)$. Unfortunately they cannot be expressed in as simple a form. However, an approximate expression for $E_{z}$ near the antenna may be obtained from

$$
B_{\theta}(\rho, z) \doteq \frac{I_{z}(z)}{2 \pi \nu_{0} \rho}, \quad E_{\rho}(\rho, z) \doteq \frac{q(z)}{2 \pi \epsilon_{0} \rho}
$$

which are valid for all distributions of current and charge. It follows from (14a) that

$$
\frac{\partial E_{z}(\rho, z)}{\partial \rho} \doteq \frac{1}{2 \pi \rho}\left[\frac{1}{\epsilon_{0}} \frac{\partial q(z)}{\partial z}+j \frac{\omega}{\nu_{0}} I_{z}(z)\right]
$$

With (49) and (51) and for $z \geqslant 0$, this leads to

$$
E_{z}(\rho, z)=\int_{a}^{\rho} \frac{\partial E_{z}}{\partial \rho} d \rho=\frac{k_{0} V_{0}^{e}}{\Psi_{d R} \cos k_{0} h}\left[T_{U} \cos k_{0} h-T_{D}\left(\frac{3}{4} \cos \frac{1}{2} k_{0} z-\cos \frac{1}{2} k_{0} h\right)\right] \ln \frac{\rho}{a} .
$$

Similarly, when $k_{0} h$ is near $\pi / 2$,

$$
E_{z}(\rho, z)=\frac{-k_{0} V_{0}^{e}}{\Psi_{d R}}\left[\sin k_{0} h+T_{U}^{\prime} \cos k_{0} h+T_{D}^{\prime}\left(\frac{3}{4} \cos \frac{1}{2} k_{0} z-\cos \frac{1}{2} k_{0} h\right)\right] \ln \frac{\rho}{a} .
$$

Note that these expressions satisfy the boundary condition $E_{z}=0$ at $\rho=a$. They are useful where the approximations, $\quad r_{1}=\sqrt{(h-z)^{2}+\rho^{2}} \doteq|h-z|, r_{2}=\sqrt{(h+z)^{2}+\rho^{2}} \doteq h+z, \quad r_{0}=\sqrt{z^{2}+\rho^{2}} \doteq|z|$ are acceptable. 
For the two antennas to which $(57 \mathrm{a}, \mathrm{b})$ apply

$$
\begin{aligned}
& k_{0} h=\frac{\pi}{2}, \frac{\lambda E_{z}(\rho, z)}{V_{0}^{e}}=-\left[0.253-j 0.018+(0.809+j 0.019) \cos \frac{1}{2} k_{0} z\right] \ln \frac{\rho}{a} \\
& k_{0} h=\pi, \frac{\lambda E_{z}(\rho, z)}{V_{0}^{e}}=\left[0.145-j 0.138+(0.102-j 0.095) \cos \frac{1}{2} k_{0} z\right] \ln \frac{\rho}{a} .
\end{aligned}
$$

For comparison with the sinusoidal theory it is convenient to use the quantity $\left|\lambda E_{z}(\rho, 0) / \zeta_{0} I_{m}\right|$. With $k_{0} h=\frac{\pi}{2}, I_{m} \doteq\left|I_{z}(0)\right|$; with $k_{0} h=\pi, I_{m} \doteq\left|I_{z}\left(\frac{\lambda}{4}\right)\right|$. The relevant quantities are

$$
\begin{gathered}
k_{0} h=\frac{\pi}{2}, \frac{\lambda E_{z}(\rho, 0)}{\zeta_{0} I_{z}(0)}=\frac{\lambda E_{z}(\rho, 0)}{V_{0}^{e}} \frac{Z_{0}}{\zeta_{0}}=-(0.234+j 0.117) \ln \frac{\rho}{a} \\
k_{0} h=\pi, \frac{\lambda E_{z}\left(\rho, \frac{\lambda}{4}\right)}{\zeta_{0} I_{z}(\lambda / 4)}=\frac{\lambda E_{z}\left(\rho, \frac{\lambda}{4}\right)}{V_{0}^{e}} \frac{V_{0}^{e}}{I_{z}(\rho) \zeta_{0}}=(-0.154+j 0.269) \ln \frac{\rho}{a} .
\end{gathered}
$$

These formulas are useful when $(\rho / h)^{2}<<1$ or $(\rho / \lambda)^{2}<<0.25$, which is precisely the region where $E_{z}$ as evaluated from the sinusoidal theory is incorrect.

A graphical comparison of $\left|\lambda E_{z}(\rho, 0) / \zeta_{0} I_{z}(0)\right|$ for the sinusoidal theory as determined from (9c) with the three-term formula (65) is shown in figure 12. The broken line, representing (65), is a fair approximation when $\frac{\rho}{a}$ is in the range from 1 to about 10 (which corresponds to $\frac{\rho}{\lambda}$ in the range from 0.007 to 0.07 to satisfy the condition $\left.\rho^{2} / \lambda^{2}=0.005 \ll 0.25\right)$. On the other hand, at larger distances $\left(\rho \sim \frac{\lambda}{2}\right)$ the fields given by the three-term theory and the sinusoidal theory should be quite comparable if $I_{z}(0)=I_{m}$ is the same. Hence, the solid-line curve in figure 12 should be a good approximation when $\frac{\rho}{a} \geqslant 50$ (corresponding to $\frac{\rho}{\lambda} \geqslant 0.35$ ). Between these ranges neither formula is correct, but it may be assumed that they join continuously and smoothly as suggested by the dotted curve.

Until an accurate evaluation of the near field of a center-driven antenna is available, a reasonable approximation of $E_{z}(\rho, z)$ is given by (62) or (63) when $\frac{\rho}{\lambda}$ is small and by (9c) when $\frac{\rho}{\lambda}$ is large. In an intermediate range an estimated smooth connection between the two theories may be made in the manner suggested in figure 12.

FIGURE 12. $\mathrm{E}_{\mathrm{z}}(\rho, 0)$ near a half-wave dipole.

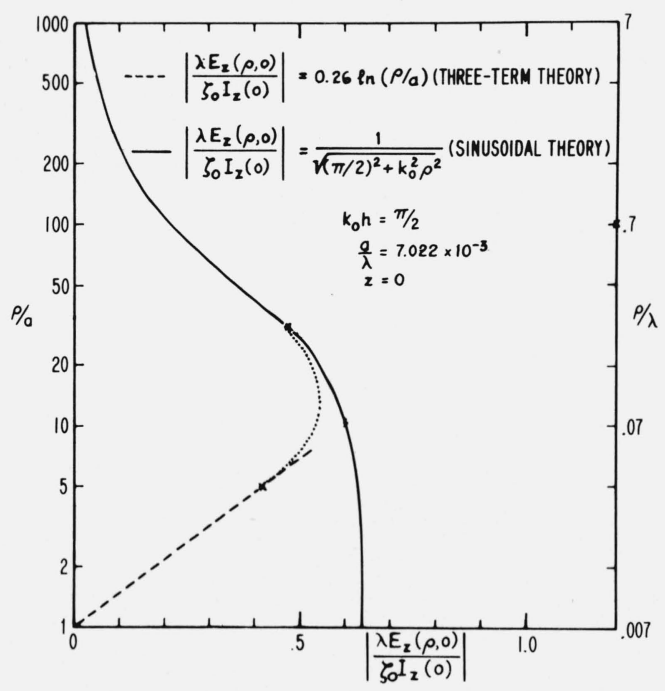




\section{Conclusion}

The assumption that the distribution of current along a center-driven highly conducting antenna is sinusoidal is inadequate to determine the distribution of charge on and the electric field near the antenna. A new relatively simple representation of the current and associated charge has been determined which is a much better approximation and which provides a means for determining the approximate near field. The results suggest that approximate solutions of dipole antenna problems that depend on an assumed sinusoidal distribution of current and involve the environment near and on the surface of a highly conducting antenna may be of questionable validity.

Note that (62) and (63) do not take into account of contributions to $E_{z}(\rho, z)$ by Coulomb-type fields near the generator at $z=0$ and near the ends at $z= \pm h$. These latter are responsible for the large peaks in figures $2 \mathrm{a}$ and $2 \mathrm{~b}$.

\section{Appendix A}

The several $\Psi$ functions introduced in $(40 \mathrm{a}-\mathrm{e})$ are defined as follows:

$$
\Psi_{d R}= \begin{cases}\Psi_{d R}(0), & k_{0} h \leqslant \pi / 2 \\ \Psi_{d R}\left(h-\frac{\lambda}{4}\right), & \pi / 2 \leqslant k_{0} h\end{cases}
$$

where

$$
\begin{aligned}
& \Psi_{d R}(z)=\csc k_{0}(h-|z|) \int_{-h}^{h} \sin k_{0}\left(h-\left|z^{\prime}\right|\right)\left[\frac{\cos k_{0} r}{r}-\frac{\cos k_{0} r_{h}}{r_{h}}\right] d z^{\prime} \\
& \Psi_{d U R}=\Psi_{d U R}(0)
\end{aligned}
$$

where

$$
\begin{aligned}
& \Psi_{d U R}(z)=\left[\cos k_{0} z-\cos k_{0} h\right]^{-1} \int_{-h}^{h}\left[\cos k_{0} z^{\prime}-\cos k_{0} h\right]\left[\frac{\cos k_{0} r}{r}-\frac{\cos k_{0} r_{h}}{r_{h}}\right] d z^{\prime} \\
& \Psi_{d D}=\Psi_{d D}(0)
\end{aligned}
$$

where

$$
\begin{aligned}
& \Psi_{d D}(z)=\left[\cos \frac{1}{2} k_{0} z-\cos \frac{1}{2} k_{0} h\right]^{-1} \int_{-h}^{h}\left[\cos \frac{1}{2} k_{0} z^{\prime}-\cos \frac{1}{2} k_{0} h\right]\left[\frac{e^{-j k_{0} r}}{r}-\frac{\mathrm{e}^{-j k_{0} r_{h}}}{r_{h}}\right] d z^{\prime} \\
& \Psi_{d I}=\Psi_{d I}(0)
\end{aligned}
$$

where

$$
\begin{aligned}
& \Psi_{d I}(z)=-\left[\cos \frac{1}{2} k_{0} z-\cos \frac{1}{2} k_{0} h\right]^{-1} \int_{-h}^{h} \sin k_{0}\left(h-\left|z^{\prime}\right|\right)\left[\frac{\sin k_{0} r}{r}-\frac{\sin k_{0} r_{h}}{r_{h}}\right] d z^{\prime} \\
& \Psi_{d U I}=\Psi_{d U I}(0)
\end{aligned}
$$

where

$$
\Psi_{d U I}(z)=-\left[\cos \frac{1}{2} k_{0} z-\cos \frac{1}{2} k_{0} h\right]^{-1} \int_{-h}^{h}\left[\cos k_{0} z^{\prime}-\cos k_{0} h\right]\left[\frac{\sin k_{0} r}{r}-\frac{\sin k_{0} r_{h}}{r_{h}}\right] d z^{\prime} .
$$


A graphical representation of the several functions $\Psi(z)$ for $k_{0} h=\frac{\pi}{2}, \frac{3 \pi}{4}$, and $\pi$ indicates that these functions are in fact reasonably constant at the indicated values $z$.

The functions $\Psi(h)$ introduced in (41) are defined as follows:

$$
\begin{aligned}
& \Psi_{V}(h)=\int_{-h}^{h} \sin k_{0}\left(h-\left|z^{\prime}\right|\right) \frac{e^{-j k_{0} r_{h}}}{r_{h}} d z^{\prime} \\
& \Psi_{U}(h)=\int_{-h}^{h}\left(\cos k_{0} z^{\prime}-\cos k_{0} h\right) \frac{e^{-j k_{0} r_{h}}}{r_{h}} d z^{\prime} \\
& \Psi_{D}(h)=\int_{-h}^{h}\left(\cos \frac{1}{2} k_{0} z^{\prime}-\cos \frac{1}{2} k_{0} h\right) \frac{e^{-j k_{0} r_{h}}}{r_{h}} d z^{\prime} .
\end{aligned}
$$

In the above formulas,

$$
r=\sqrt{\left(z-z^{\prime}\right)^{2}+a^{2}}, \quad r_{h}=\sqrt{\left(h-z^{\prime}\right)^{2}+a^{2}} .
$$

All of the above integrals can be expressed in terms of the tabulated generalized sine and cosine integrals [Staff of Computation Laboratory, 1949] without difficulty except the following one which involves some manipulation:

$$
\begin{aligned}
\int_{-h}^{h} \cos \frac{1}{2} k_{0} z^{\prime} \frac{e^{-j k_{0} r}}{r} d z^{\prime}=\left[\sinh ^{-1} \frac{h+z}{a}+\sinh ^{-1} \frac{h-z}{a}\right] \cos \frac{1}{2} k_{0} z \\
-\frac{1}{2} e^{j k_{0} z / 2}\left[C\left(A^{\prime}, \frac{1}{2} k_{0}(h-z)\right)+C\left(A^{\prime}, \frac{3}{2} k_{0}(h+z)\right)+j S\left(A^{\prime}, \frac{1}{2} k_{0}(h-z)\right)+j S\left(A^{\prime}, \frac{3}{2} k_{0}(h+z)\right)\right] \\
-\frac{1}{2} e^{-j k_{0} z / 2}\left[C\left(A^{\prime}, \frac{1}{2} k_{0}(h+z)\right)+C\left(A^{\prime}, \frac{3}{2} k_{0}(h-z)\right)+j S\left(A^{\prime}, \frac{1}{2} k_{0}(h+z)\right)+j S\left(A^{\prime}, \frac{3}{2} k_{0}(h-z)\right)\right]
\end{aligned}
$$

where $A^{\prime}=\left(k_{0} a \sqrt{3}\right) / 2, C$ and $S$ are tabulated generalized cosine and sine integral functions. They are defined as follows:

$$
\begin{aligned}
& S(a, x)=\int_{0}^{x} \frac{\sin W}{W} d u \\
& C(a, x)=\int_{0}^{x} \frac{1-\cos W}{W} d u
\end{aligned}
$$

where $W=\sqrt{u^{2}+a^{2}}$.

\section{Appendix B}

The coefficient $T_{U}^{\prime}$ is defined in (52b) with (48a). It may be expressed as follows:

$$
T_{U}^{\prime}=\frac{\Psi_{d D} \Psi_{V}(h)-j \Psi_{d I} \Psi_{D}(h)+Q \sin k_{0} h}{Q \cos k_{0} h}
$$

where

$$
Q=\Psi_{d D}\left[\Psi_{d U R} \cos k_{0} h-\Psi_{U}(h)\right]+j \Psi_{d U I} \Psi_{D}(h)
$$

It is readily seen from $(\mathrm{A}-1),(\mathrm{A}-2),(\mathrm{A}-4),(\mathrm{A}-5),(\mathrm{A}-6)$, and $(\mathrm{A}-7)$ that when $k_{0} h=\frac{\pi}{2}, \Psi_{d U R}=\Psi_{d R}$, 
$\Psi_{d U I}=\Psi_{d I}, \Psi_{U}(h)=\Psi_{V}(h)$. It follows that

$$
T_{U}^{\prime}=\frac{\Psi_{d D} \Psi_{d R}}{\Psi_{d D} \Psi_{V}(h)-j \Psi_{d I} \Psi_{D}(h)}, \quad h=\frac{\lambda}{4} .
$$

Similarly, $T_{D}^{\prime}$ as defined in (63) with $(47 \mathrm{~b})$ is given by

$$
T_{D}^{\prime}=\frac{-j\left\{\Psi_{d I}\left[\Psi_{d U R} \cos k_{0} h-\Psi_{U}(h)\right]+\Psi_{d U I} \Psi_{V}(h)\right\}}{Q \cos k_{0} h} .
$$

When $k_{0} h=\frac{\pi}{2}$, this reduces to

$$
T_{D}^{\prime}=\frac{j \Psi_{d I} \Psi_{d U R}}{\Psi_{d D} \Psi_{V}(h)-j \Psi_{d I} \Psi_{D}(h)}, \quad h=\frac{\lambda}{4}
$$

\section{References}

Ament, W. S., J. C. Katzin, M. Katzin, and B. Y.-C. Koo (1964), Impedance of a cylindrical dipole having a sinusoidal current distribution in a homogeneous anisotropic ionsophere, Radio Sci. J. Res. NBS/USNC-URSI 68D, No. 4, $379-405$.

Hallén, E. (1938), Theoretical investigations into transmitting and receiving antennae, Nova Acta Regia Soc. Sci. Upsaliensis, Ser. 4, No. 11, 1.

King, R. W. P. (1955a), Transmission-line theory, p. 70 (McGraw-Hill Book Co., Inc., New York, N.Y.).

King, R. W. P. (1955b), Transmission-line theory, p. 368 (McGraw-Hill Book Co., Inc., New York, N.Y.).

King, R. W. P. (1956a), Theory of linear antennas, pp. 525-528 (Harvard University Press, Cambridge, Mass.).

King, R. W. P. (1956b), Theory of linear antennas, ch. 2 (Harvard University Press, Cambridge, Mass.).

King, R. W. P. (1959), Linear arrays: Current, impedances, and fields, I. (Harvard University, Cambridge, Mass., May 1, 1959) Trans. IRE AP-7, 5440.

King, R., and C. W. Harrison, Jr. (1943), The distribution of current along a symmetrical center-driven antenna, Proc. IRE 31,548 .

Mack, R. B. (1964), A study of circular arrays, parts 2 and 3, Technical Report Nos. 382 and 383. Cruft Laboratory, Harvard University, Cambridge, Mass.

Staff of Computation Laboratory (1949), Tables of generalized sine- and cosine-integral functions, parts 1 and 2 (Harvard University Press, Cambridge, Mass.). (In abbreviated form in the appendix King, Theory of linear antennas, Harvard University Press, 1956.)

Storer, J. E. (1951), Variational solution to the problem of the symmetrical cylindrical antenna, Cruft Lab. Tech. Report No. 101, Harvard University, Cambridge, Mass. (Also in King, 1956b.)

Tai, C. T. (1950), A variational solution to the problem of cylindrical antennas, Tech. Report No. 12, SRI Project No. 188, Stanford Research Institute, Stanford, Calif. (Also in King 1956b.)

(Paper 69D3-481) 